\title{
aufbruch - Forum für eine offene Kirche \\ Identitätssuche einer jungen ökumenischen Zeitung in der Spannung zwischen Option und Erfolg
}

von Matthias Müller

Ich nenne euch nicht mehr Knechte; denn der Knecht weiß nicht, was sein Herr tut. Vielmehr habe ich euch Freunde genannt; denn ich habe euch alles mitgeteilt, was ich von meinem Vater gehört habe. Joh 15,15

\section{Einleitung}

Wer die jüngere Entwicklung des Kommunikationsverständnisses in der Lehre der katholischen Kirche in einer prägnanten Form zusammenfassen will, findet im obigen Vers aus dem Johannes-Evangelium eine adäquate biblische Ausdrucksweise. Diese Aussage muß aber sogleich relativiert werden, denn von dem, was im Zuge des Zweiten Vatikanischen Konzils diesbezüglich auf theoretischer Ebene aufgebrochen ist, ist in den vergangenen dreißig Jahren nur ein Bruchteil spürbar und dauerhaft zum Tragen gekommen. Daher müßte man korrekter sagen: Der obige Vers könnte als ,Leitspruch' über einer wünschenswerten kirchlichen Kommunikationskultur stehen.

Diese Ambivalenz zwischen theoretisch vorhandenem Bewußtsein in der Lehre der Kirche und dem faktischen Unvermögen bzw. dem Unwillen vieler Amtsinhaber, adäquat mit den Erkenntnissen im Bereich der Kommunikation, mit ihren Formen und Mitteln umzugehen, führt dazu, daß die Kirche besonders in Konfliktsituationen ein oft zwiespältiges Bild nach außen vermittelt. Kirchliche Konflikte bringen oft nicht nur Mißstände in den inneren Strukturen an den Tag, sondern werden immer stärker auch zu Prüfsteinen für eine kirchliche Kommunikationskultur.

Die schweizerische ökumenische Zeitung aufbruch - Forum für eine offene Kirche wurde 1988 als Reaktion auf den sogenannten ,Fall Haas', den Konflikt im Bistum Chur um die Einsetzung von Wolfgang Haas zum Weihbischof mit Nachfolgerecht, lanciert. Bezeichnend für den Verlauf des Konflikts war, daß die theologischen Streitpunkte verhält-

Matthias Müller studiert Journalistik und Theologie an der Universität Fribourg/Schweiz. 
nismäßig rasch an Bedeutung verloren, während die Frage nach einer gesunden, der Zusammenarbeit von Basis und Bistumsleitung förderlichen Kommunikationskultur immer dringlicher wurde. In der Auseinandersetzung prallten im Grunde weniger die Kirchenleitung und die Basis, sondern vielmehr zwei verschiedene Kirchenbilder aufeinander, die ihre Wurzeln in unterschiedlichen Offenbarungsverständnissen haben und sich entsprechend in ungleichen Kommunikationsverständnissen artikulieren.

Weil sich neben der ungewöhnlichen Entstehungsgeschichte des aufbruch auch seine ganze weitere Entwicklung und die darin eingeschlossene Identitätssuche nur auf dem ihn umgebenden kirchlichreligiösen Umfeld verstehen läßt, werde ich in einem ersten Teil nach einer knappen Skizzierung des ,Falles Haas' auch den kommunikationstheoretisch-theologischen Hintergrund beleuchten.

Als der aufbruch im Februar 1995 seine 50. Ausgabe feiern konnte, schaute die Redaktion auf sieben bewegte Jahre zurück - und gleichzeitig einer unsicheren Zukunft entgegen: Dem anfänglich außerordentlichen Erfolg der Zeitung folgten empfindlich sinkende Abonnementszahlen. Verbunden mit dem in der Zwischenzeit stark veränderten kirchlichen Umfeld machte dies eine intensive Neuorientierungsphase nötig, die nicht zuletzt von der Spannung zwischen ,Option' und ,Erfolg' - der Frage nach dem Überleben - geprägt war und nach wie vor ist. Die konkrete Entstehung des aufbruch, seine Entwicklung und schließlich seine aktuelle Situation bilden den zweiten Teil des Artikels. Darin werden auch Ergebnisse der LeserInnenumfrage aufgenommen, die ich im Februar 1995 im Auftrag der Redaktion durchführte.

\section{Kommunikations(un-)kultur im "Fall Haas"}

Der sogenannte ,Fall Haas‘ gehört zu denjenigen kirchlichen Konflikten, die in den letzten Jahren auf großes ,säkulares' Medieninteresse gestoßen sind. In dieselbe Linie lassen sich beispielsweise der Wirbel um Bischof Gröer in Wien oder die Absetzung von Bischof Gaillot (Evreux/Frankreich) einreihen. Alle diese Konflikte zeigen ein ähnliches Muster: Sie waren geprägt von der Auseinandersetzung zweier Kirchenbilder, die unter anderem je ihre deutlicher oder weniger deutlich ausgeprägte, mehr oder minder getrennte Vorstellung von, interner kirchlicher Kommunikation' und ,sozialer Kommunikation' haben. Die jeweils an den Tag gelegte, interne kirchliche Kommunikationskultur', die oft ein Mittel zur Klärung oder gar Bewältigung des Konflikts hätte darstellen können, ließ stark zu wünschen übrig.

Als im April 1988 der damalige Kanzler des Bistums Chur, Wolfgang Haas, auf Wunsch des amtierenden Bischofs Johannes Vonderach von Papst Johannes Paul II. zum Weihbischof mit Nachfolgerecht (Koadjutor) ernannt wurde, löste dies unter den Seelsorgerinnen 
und Seelsorgern sowie bei vielen Gläubigen einen Strum der Empörung sowie große Trauer und Bestürzung aus. Die Krise, die sich im Bistum Chur abzuzeichnen begann, weitete sich rasch auf die ganze Kirche der Schweiz aus. Es waren mehrere ineinandergreifende Faktoren, die nicht nur einzelne Personen, sondern auch ganze Pfarreien dazu veranlaßten, offen gegen die Amtseinsetzung und -ausübung von Wolfgang Haas Widerstand zu leisten: Die Art und Weise der Amtseinsetzung, bei der wegen der Übergehung des mitspracheberechtigten Domkapitels die Frage nach der Rechtmäßigkeit im Zentrum stand, sodann die eigentliche Person von Wolfgang Haas, der als Vertreter einer konservativen, zentralistisch-romtreuen Linie galt, und schließlich sein persönliches Verhalten im Amt als Weihbischof und später, nach 1990, als Diözesanbischof, in welchem er sich gegenüber den Seelsorgerinnen und Seelsorgern sowie gegenüber den Medien als kommunikationsunfähig und -unwillig erwies. Gesprächsverweigerung, Intransparenz bei Personalentscheidungen sowie Schweigen prägten auf der Seite der Bistumsleitung das Bild des Konflikts. ${ }^{1}$

Im Konflikt gelangte offen an die Oberfläche, was seit Jahren als schwelende Spannung in der katholischen Kirche zu beobachten war: Es prallten zwei Kirchenbilder aufeinander, das eine traditionell verankerte, vorkonziliare, das von Wolfang Haas verkörpert wurde, und das andere im Zweiten Vatikanischen Konzil grundgelegte, das im Bewußtsein vieler Seelsorgerinnen, Seelsorger und Gläubigen im Bistum fest verankert war. Der anfänglich immer wieder angestrebte Dialog zwischen verschiedenen Gremien bzw. Gruppen und der Bistumsleitung scheiterte meistens schon daran, daß die elementarsten Grundbedingungen für eine Verständigung fehlten: Die beiden Kirchenbilder, die sich in ihrem Wert- und Normengefüge und damit auch in entsprechenden Verhaltenserwartungen unterschieden, artikulierten sich unter anderem auch in unterschiedlichen Sprachen und Kommunikationsmodellen. Eine Verständigung als Ergebnis einer erfolgreich ablaufenden kommunikativen Interaktion mußte deshalb in der herrschenden Konfliktsituation unweigerlich scheitern. ${ }^{2}$ Obschon das Verhalten von Bischof Haas also weniger ein persönlich bedingtes als vielmehr Ausdruck eines weltweiten Problems der katholischen Kirche war, dessen Wurzeln in ihrer Innenstruktur liegen, ${ }^{3}$ mußte der Konflikt an

1 Eine Fundgrube mit einer Reihe von dokumentierten Beispielen sind die beiden Bände "Dokumentation zum Fall Haas. Band $1+2$ “, hg.v. B. Bühlmann, Luzern 1988 bzw. 1991.

Aus journalistischer Sicht besonders interessant ist, daß Wolfgang Haas 1991 den Posten des offiziellen Informationsbeauftragten des Bistums als "vakant" erklärte, nachdem der Informationsfluß von der Bistumsleitung fast drei Jahre lang am Informationsbeauftragten vorbeigelaufen war und dieser das Neueste jeweils aus den Zeitungen erfahren hatte.

2 Vgl. dazu R. Burkhart, Kommunikationswissenschaft, Wien 1983, 93.

3 Vgl. A. Ricklin, Bischofswahlen in der Schweiz, in: Schweizerische Kirchenzeitung (SKZ), 19.05.94, 300. 
seiner Person eskalieren, "weil er - menschlich zuvorkommend und freundlich selbst erbittertsten Gegnern gegenüber - in der Sache notorisch und unverrückbar die Positionen ausschließlich des restaurativ-zentralistischen, nur kirchenrechtlich orientierten Segments der Kirche vertritt. Echter Dialog wird so unmöglich: Wohl ,hört' er, wenn nötig, stundenlang zu, doch irgendwie scheinen Empfindungen und Argumente an ihm abzuprallen wie an einem Regenmantel: Die zutiefst verletzten und letztlich nicht ernstgenommenen (...) Seelsorger kommen sich vor, als wollten sie Magnete an einer Plastikwand zum Haften bringen - doch das kann nicht gelingen ". 4

\section{Instruktion versus Kommunikation}

Die im Konflikt aufeinanderprallenden Vorstellungen, wie die innerkirchliche Kommunikation aussehen sollte, beruhen auf den unterschiedlichen Offenbarungsverständnissen, die ihnen zugrunde liegen und die jeweils zu unterschiedlichen Kirchenbildern führen. Sie soilen beide in ihren markantesten Zügen dargestellt werden.

Auf der einen Seite steht das von der Mitte des 19. Jahrhunderts bis zum Zweiten Vatikanischen Konzil gelehrte ,instruktionstheoretische Offenbarungsmodell', in welchem die Belehrung des Menschen durch Gott auf übernatürlichem Wege vor sich geht: In einem von der christlichen Mythologie geprägten Bild steigt Jesus Christus vom Himmel herab auf die Erde und bringt die göttliche Lehre mit. Nach seinem Wirken und der Rückkehr in den Himmel wird diese Lehre durch das Lehramt gehütet und weitergegeben. Dieses ist bezüglich des Weitergebens dieser Sätze unfehlbar. Innerkirchliche Kommunikation beschränkt sich auf die authentische und unverfälschte Weitergabe des Glaubensgutes und verläuft linear-einseitig. Die ,Rückmeldung' in Form einer moralischen Kontrolle (Beichte) dient nicht zuletzt der Machterhaltung des Lehramtes. Der Glaube besteht in einem Für-wahr-Halten der vom Lehramt vermittelten Sätze. Die, weltlichen Medien' werden in diesem Kirchenbild der Einheitlichkeit, Zentralisierung, Disziplinierung 5 sowie der Abgrenzung gegen unten und des Gehorsams gegenüber der

4 G. Rimann, Kann Kirche auf Dauer so wirklich leben?, in: M. Amherd (Hg.), Wolfgang Haas: Bischof ohne Volk - Volk ohne Bischof, Zürich 1991, 45f.

5 Es ist nicht untypisch, daß die katholische Kirche primär mit dem Medium der geschriebenen Sprache arbeitet: „Systeme wie das Römische Reich oder die Katholische Kirche wären ohne das regulative Potential der Schriftsprache so nicht denkbar gewesen. Gesetzestexte, Verwaltungsnormen und Hirtenbriefe stellen eine Einheitlichkeit der regulativen Verhaltensformen sicher (...). Noch heute können wir am Umgang der Katholischen Kirche mit Texten - und Textverboten - beobachten, wie sehr sich diese Institution der regulativen Funktion der Schriftsprache bewußt ist." (K. Böckmann, Unser Weltbild aus Zeichen. Zur Theorie der Kommunikationsmedien, Wien 1994, 180). 
Obrigkeit oft nur funktional als im eigenen Dienst stehende Hilfsmittel gesehen. Eine adäquate Auseinandersetzung mit ihnen findet kaum statt, und im extremsten Fall können sie sogar zu einem Bestandteil des Feindbildes ,Moderne' werden.

Dem stellt das Zweiten Vatikanischen Konzil ein verändertes ekklesiologische Modell entgegen, das seinerseits auf einem veränderten Offenbarungsverständnis aufbaut: Das ,kommunikationstheoretische Offenbarungsmodell' geht davon aus, daß die Offenbarung Gottes in "Wort und Tat"6 erfolgt. Den Höhepunkt und die Vollendung erreichte die Offenbarung im Sichselbstmitteilen Gottes in Jesus von Nazareth (Tat). Diese ist uns heute noch unter anderem in Form der biblischen Zeugnisse als ,Wort' zugänglich. Theologische Wahrheiten werden analog zu ihrer Überlieferung durch die Zeit - über einen kommunikativen Prozeß rückerschlossen. Dieser Weg steht prinzipiell allen Gläubigen offen. Im Kirchenbild schlägt sich dies in Form der neuen Terminologie des , pilgernden Gottesvolkes' nieder. Neben der weiterhin starken hierarchischen Struktur soll damit die horizontale Verbundenheit aller Christinnen und Christen (den Klerus eingeschlossen) als ein Volk stärker betont werden. Anstelle des Übereinander tritt das Mitund Füreinander. Dieses Kirchenbild der Geschwisterlichkeit und der dialogisch verfaßten Gemeinschaft vertraut auf die aktive, mündige Teilnahme aller Christen. Die Kirche erscheint „als eine universale Kommunikationsgemeinschaft, in der über die Glaubwürdigkeit bestimmter kirchlicher Auffassungen und Interpretationen im Sinne eines auf Konsensbildung ausgerichteten Prozesses verhandelt wird. Die Kritik am juridisch-hierarchischen Kirchenverständnis ist von dieser Position aus nicht zufällig, sondern konstitutiv mit ihr verbunden: indem das hierarchische Kirchenverständnis das Problem der Rezeption auf die Gehorsamsbereitschaft reduziert, werden schon die Voraussetzungen eines offenen innerkirchlichen Kommunikationsprozesses, nämlich die Anerkennung der Rezeptionsbedürftigkeit negiert. ${ }^{\text {7 }}$

\section{Theoretisches Bewußtsein in den Dokumenten}

Im Rahmen des ,aggiornamento' des Zweiten Vatikanischen Konzils begann die Amtskirche, auch ihr allgemeines Verhältnis zu den Medien systematisch zu reflektieren. Das Dokument "Inter mirifica“ (1963), das im Licht des ganzen Konzils jedoch noch als Frühgeburt zu bezeichnen

6 Vgl. Konzilstext ,Dei verbum' 2, in: K. Rahner/H. Vorgrimler (Hg.), Kleines Konzilskompendium, Sämtliche Texte des Zweiten Vatikanums mit Einführungen und ausführlichem Sachregister, Freiburg i.Br. ${ }^{25} 1994,368$.

7 F.-X. Kaufmann, Glaube und Kommunikation, in: D. Wiederkehr (Hg.), Der Glaubenssinn des Gottesvolkes - Konkurrent oder Partner des Lehramtes?, Freiburg i.Br. 1994, 143. 
ist, ${ }^{8}$ hat als ein erster Ausdruck davon die sozialen Kommunikationsmittel ins Zentrum kirchlicher Reflexion gerückt. Dabei wurde zwar das Informationsrecht in der menschlichen Gesellschaft nachdrücklich betont, das Recht auf Information in der Kirche wurde jedoch mit Schweigen übergangen. ${ }^{9}$ Auch wenn die späteren kirchlichen Dokumente wie "Gaudium und spes" (1965), "Communio et progressio" (1971) oder dessen Nachfolgedokument „Aetatis novae" (1992) zu einem stetig sich verändernden theoretischen Bewußtsein der Kirche in ihrer Auseinandersetzung mit den sozialen Kommunikationsmitteln beigetragen haben, so steht dies heute doch oft in Widerspruch zur realen Praxis der Amtskirche. Das Resultat davon ist ein unstimmiges Bild nach außen: „Zwar gibt es durchaus kirchliche Dokumente (...), welche die Bedeutung der sozialen Kommunikationsmittel sehr wohl erkennen und auch die Eigengesetzlichkeiten der Massenmedien respektieren. In der Praxis allerdings ist wenig von einer offenen und angstfreien Kommunikationskultur der Kirche zu spüren. Vielmehr zeigt sich, daß Kommunikationsunfähigkeit nirgends so deutlich zum Ausdruck kommt wie in der Kirche selbst." ${ }^{10}$

\section{Sonderfall Schweiz}

In der Schweiz ließ man den Geist des Konzils auf diözesaner Ebene in den Beratungen der synodalen Versammlungen weiterleben. Das Ziel war, den Konzilsbegriff "Kirche am Ort ${ }^{11}$ auch in der ,Kirche Schweiz' mit ihren spezifischen Ausprägungen voll zum Tragen bringen zu lassen. Im Bistum Chur resultierten daraus die Texte der sogenannten ,Synode 72'.

Die nachkonziliaren Bewegungen haben in der Schweiz vor allem im pastoralen Bereich entscheidende Veränderungen zugunsten der Mündigkeit der Gläubigen gebracht, wobei man bei der Umsetzung der Konzilsimpulse, verglichen mit den umliegenden Ländern, gewisse Sonderwege einschlug - mit mehr oder minder wohlwollend erteiltem Segen oder zumindest widerspruchslosem Gewährenlassen Roms. ${ }^{12}$

Diese Neuerungen führten in den folgenden Jahren zu einem veränderten und vor allem gestärkten Bewußtsein unter den Gläubigen. Die Ernennung von Wolfgang Haas zum Weihbischof mit Nachfolge-

8 Vgl. Einleitung zu "Inter mirifica”, in: K. Rahner/H. Vorgrimler (Hg.), Kleines Konzilskompendium (Anm. 6), $91 \mathrm{f}$.

9 Vgl. ebd.

10 B. Bühlmann, Anwaltschaftlicher Journalismus?, in: Theologische Bewegung für Solidarität und Befreiung (Hg.), Rundbrief Nr. 38, Luzern 1993.

11 Vgl. die beiden Konzilstexte: Lumen gentium 23 und Orientalium ecclesiarum 4, in: K. Rahner/H. Vorgrimler (Hg.), Kleines Konzilskompendium (Anm. 6), 149ff bzw. 206f.

12 Vgl. G. Rimann, Kann Kirche auf Dauer so wirklich leben? (Anm. 4), 47. 
recht im April 1988 förderte deshalb in den Augen der Seelsorgerinnen und Seelsorger "eine gefährliche Resignation und ist nicht zuletzt ein Verlust von Autorität und Glaubwürdigkeit für die Kirche bei den Menschen, die ermutigt - durch Konzil und Synode 72 - begonnen haben, Kirche aktiv und kritisch mitzutragen und mitzugestalten" ${ }^{13}$

Die kirchenpolitischen, kirchenrechtlichen und theologischen Differenzen, die sich im ,Fall Haas' zeigten, wurden somit durch den spezifischen Kontext der ,Kirche Schweiz' zusätzlich verstärkt.

\section{Der Aufbruch der Basis}

Die Ereignisse vom April 1988 und der folgenden Monate rissen wilde Furchen in diesen in gewisser Hinsicht speziell ausgeprägten schweizerischen ,Glaubensacker' und zogen ihn damit arg in Mitleidenschaft. Gleichzeitig wurde er aber zum Nährboden für einen zunächst bistumsinternen, bald aber gesamtschweizerischen Aufbruch innerhalb der Kirche Schweiz, der sich in Form eines für die katholische Kirche ungewohnt breiten, offenen und direkten Widerstandes zeigte: Nachdem Protestbriefaktionen, Aktionsgruppen, Bittschriften und ähnliches die Weihe von Wolfgang Haas zum Bischof am 22. Mai 1988 nicht verhindern konnten, fand am Vortag in Zürich eine Solidaritätskundgebung für eine, weltoffene, mündige und befreiende Kirche' statt. In einer Postkartenaktion wurden alle Teilnehmerinnen und Teilnehmer aufgerufen, Ideen und Anregungen zu formulieren, wie die Anliegen des Zweiten Vatikanischen Konzils und der Synode 72 weitergetragen werden können. Einig war man sich bereits darüber, daß die solidarischen Kräfte der kirchlichen ,Basis' weiterhin und über die Bistumsgrenzen hinaus zusammengehalten werden müssen. Um diese Verbundenheit zu stärken, kamen am 19. Juni 1988 ca. sechzig Frauen und Männer in Luzern zu einem ,Schulterschluß an der Basis' zusammen. Sie suchten weitere Schritte, die zu einer ,weltoffenen, mündigen und befreienden Kirche führen sollten. „Was (...) von ziemlich allen Anwesenden am Schluß des Treffens in Kurzvoten geäußert wurde, ist die Notwendigkeit einer ,Bewegung' der Kirchenbasis im Sinne der Anliegen und der Verpflichtung von Konzil und Synode. Auch eine Zeitung als Drehscheibe gehört zu den nächsten Zielen. Am 20. August (...) werden in der nächsten Sitzung (...) konkrete Schritte für diese Sammelbewegung in die Wege geleitet. Dazu hat sich eine Kerngruppe formiert [mit dem Namen ,Basiskirchliche Vernetzung' BKV; d. Verf.], wobei aber natürlich wieder alle Interessierten und

13 Aus: "Offene Stellungnahme von Priestern, Ordensleuten, Mitarbeiterinnen und Mitarbeitern in Seelsorge und Verkündigung des Bistums Chur" von Anfang April 1988, in: B. Bühlmann ( $\mathrm{Hg}$.), Dokumentation zum Fall Haas, Band 1 (Anm. 1), $13 \mathrm{f}$. 
Betroffenen herzlich willkommen und eingeladen sind mitzutragen." ${ }^{14}$ Damit war die Idee einer landesweiten Basisbewegung mit einer Art Mitgliedszeitschrift als Sprachrohr grundgelegt.

\section{Eine Zeitung wird geboren}

Was im Fazit dieses Treffens verhalten als "auch eine Zeitung" formuliert wurde, ging nur fünf Monate später als "aufbruch Nr. 1 " in einer Startauflage von 100.000 Exemplaren über die Rotationsmaschine einer Winterthurer Druckerei. Die eigentliche, konkrete Lancierungsphase der Zeitung begann jedoch erst Ende September 1988: Auf einer Sitzung entschied die BKV-Untergruppe für Öffentlichkeitsarbeit, bis Anfang Dezember 1988 eine Zeitung mit dem Namen „aufbruch - Forum für eine offene Kirche" herauszugeben, die als Sprachrohr und Sammelpunkt zur basiskirchlichen Vernetzung beitragen soll. Es wurde ein grobes Konzept zusammengestellt und der Inhalt der ersten Nummer abgesprochen. ${ }^{15}$

Nur knapp vier Wochen später sollte in Zusammenarbeit mit einem Grafiker eine Nullnummer vorliegen, von der ausgehend man das genaue Zeitungsprojekt beschließen konnte. Wieder nur zehn Tage später, am 2. November, fand die eigentliche Redaktionssitzung für die erste aufbruch-Ausgabe statt. Gleichzeitig gründete man den Verein ,Förderkreis aufbruch', der als juristische Trägerschaft der Zeitung auftreten sollte. Bis zum Erscheinen der ersten Nummer Ende November 1988 waren dem Verein bereits zehn basiskirchliche Gruppierungen und etwa 100 Einzelpersonen beigetreten, die die Zeitung finanziell und ideell mittrugen und deren unmittelbare Zukunft sicherten.

Den 28. November 1988 kann man als Geburtstag des aufbruch bezeichnen: Nach dem Druckprozeß wurde er im Rahmen einer Pressekonferenz in Zürich rund 30 Journalisten vorgestellt.

\section{Das Leitbild}

Das Leitbild des aufbruch wurde wie folgt umschrieben:

- „Die Motivation für unseren AUFBRUCH ist die Vision von einer wahrhaft menschlichen, geschwisterlichen Kirche. Einer mutigen und

14 Unveröffentlichter Brief der „Initiativgruppe für einen Schweizerischen Basiskirchentag" vom 27. Juni 1988 (Hervorhebung durch den Verfasser) in: M. Müller, 50 Nummern aufbruch - Forum für eine offene Kirche. Ein Blick zurück - Eine LeserInnenumfrage - Eine Standortbestimmung. Diplomarbeit am Institut für Journalistik und Kommunikationswissenschaften der Universität Freiburg i.Ue, 1995, Anhang X.

15 Unveröffentlichtes Konzept "Zeitungsprojekt ,Forum für eine offene Kirche"', in: M. Müller, 50 Nummern auforuch (Anm. 14), Anhang XI. 
angstfreien Kirche. Diese Kirche ist heute aber in Gefahr. Angst, Vorsicht, Autoritarismus beginnen wieder, das Feld zu beherrschen.

Darin sehen wir nicht den Geist des befreienden Evangeliums.

- Den Geist des Evangeliums sehen wir hingegen eingefangen im folgenden Satz des kirchlichen Rechtsbuches von 1984: ,Entsprechend dem Wissen, der Sachkompetenz und der Stellung, die sie einnehmen, steht ihnen (den Laien) das Recht, gelegentlich auch die Pflicht $\mathrm{zu}$, den geweihten Hirten gegenüber ihre Meinung zu äußern in Fragen, die das Allgemeinwohl der Kirchen betreffen, und diese Meinung auch den übrigen Mitchristen bekannt zu machen.' (Can 212 \& 3) - Dieses Recht und diese Pflicht will AUFBRUCH unerschrocken wahrnehmen.

- AUFBRUCH will der schleichenden Resignation entgegenwirken, den klammheimlichen Auszug vieler aus der Kirche stoppen, die aufbrechenden Kräfte an der Basis miteinander ins Gespräch bringen, die basiskirchliche Vernetzung fördern und $\mathrm{zu}$ einer „Konfliktkultur' aus dem Glauben beitragen.

- AUFBRUCH sucht die konstruktive Auseinandersetzung mit andersdenkenden kirchlichen Kräften. Er will für einen transparenten Prozeß der Ökumene Hör- und Gesprächsorgan sein. Er will als Drehscheibe die vielfältigen Aufbrüche, Versuche und Aktionen an der Basis - lokal, regional und national - sammeln, koordinieren und verbreiten.

- AUFBRUCH will Mut, Hoffnung und Freude wecken, die Botschaft Jesu heute zu leben, ohne sich von starren und unbeweglichen Strukturen einschüchtern zu lassen." 16

$\mathrm{Da}$ in den Leitlinien unter anderem ein Paragraph aus dem katholischen Kirchenrecht zitiert wurde, mag als kleines Detail erscheinen. Im Kontext der Churer Ereignisse kam dem indes ein höherer Stellenwert zu: In Anlehnung an das Verhalten von Wolfgang Haas, der sich bei der Legitimierung seiner Ernennung und der Art seiner Amtsausübung stets auf geltendes kanonisches Recht berief, sollte mit Can 212 \& 3 CIC unterstrichen werden, daß die Initiative des aufbruch mindestens ebenso juristisch gesichert' war. Angriffen aus dem konservativen, dem Auftreten des aufbruch kritisch eingestellten Lager, war somit weniger Angriffsfläche geboten.

Mit dem anfänglich zweimonatlich erscheinenden aufbruch hatten sich die fortschrittlichen Kreise in der katholischen Kirche ihr eigenes Sprachrohr geschaffen. Die neue Zeitung sollte mit ihrem christlich-

16 Aus den Unterlagen zur Pressekonferenz vom 28.11.88 in Zürich, in: $M$. Müller, 50 Nummern aufbruch (Anm. 14), Anhang XIV. 
kirchlich engagierten Journalismus das faktisch bestehende Meinungsmonopol in der katholischen Presselandschaft aufbrechen, das bisher entweder von den kirchlich finanzierten Pfarrblättern oder konservativen Blättern wie der ,Katholischen Wochenzeitung dominiert wurde. ${ }^{17}$ Im weiteren wiesen die Initiatorinnen und Initiatoren von Anfang an deutlich darauf hin, daß die Zeitung nicht einzig am Churer Konflikt aufgehängt werden soll, obschon dieser den Anlaß zum Projekt gegeben hatte. ${ }^{18}$ Dies sollte für den Prozeß der Identitätsfindung während der kommenden Jahre wichtige Konsequenzen nach sich ziehen.

\section{Der aufbruch etabliert sich}

\subsection{Eindrücklicher Abonnentenzuwachs und ökumenische Öffnung}

Um den aufbruch nach seinem Erscheinen rasch in weiten Kreisen bekannt zu machen, hatte die Initiativgruppe bereits zeitig damit begonnen, Adressen von potentiell Interessierten zu sammeln. Von den 100.000 Exemplaren der ersten Nummer wurden so etwa 10.000 gezielt versandt. Zudem wurden in zehn ausgewählten ,katholischen' Ortschaften, unter ihnen Einsiedeln, Schwyz und Immensee, alle Haushaltungen beliefert. Die weitere Werbung lief primär von Mund-zu-Mund sowie über den Aufruf an die Leserinnen und Leser, der Redaktion Listen von Adressen zu senden, denen ein Probeexemplar zugestellt werden könnte. Auf diese Weise zählte der aufbruch bereits nach der dritten Nummer im Mai 1989 über 7.500 Abonnentinnen und Abonnenten, und an seinem ersten Geburtstag war die 10.000er-Grenze durchbrochen Fachleute setzten nach vergleichbaren Erfahrungen in Deutschland die Expansionslimite für den aufbruch bei rund 4.000 Abonnenten an. ${ }^{19}$

$\mathrm{Zu}$ dieser beeindruckenden Entwicklung der Abonnentenzahl im ersten Jahr lief parallel eine formelle und inhaltliche Konsolidierung: Erweiterung der Anzahl der Seiten von 12 auf 16 sowie ein erleichterter Zahlungsverkehr aus Deutschland und Österreich, womit die Redaktion dem Interesse aus dem deutschsprachigen Ausland entgegenkam.

Von größter Bedeutung war jedoch die ökumenische Öffnung, die Mitte 1989 mit der Erweiterung des Redaktionsteams um zwei evangelisch-reformierte Mitglieder vollzogen wurde. Es sollte damit unterstrichen werden, daß sich der aufbruch trotz seiner, innerkatholischen Wurzeln' an einer größeren ökumenischen Vision orientierte und $\mathrm{da}$ er eine Forderung von Konzil und Synode ernst nehmen wollte, "alles gemeinsam zu tun, was jetzt schon gemeinsam getan werden

17 Vgl. "Luzerner Neuste Nachrichten” sowie „Bündner Zeitung” vom 29.11.88.

18 Vgl. "Tages-Anzeiger" vom 29.11.88.

19 Vgl. aufbruch Nr. 3, Mai 1989, 2. Dieser Vergleich stützt sich auf die Erfahrungen mit ,Publik-Forum'. 
kann".20 Das Ziel sollte jedoch nicht sein, auf eine "profil- und geschichtslose Super-Kirche hinzuwirken. Die Grundlagen unserer Reflexionen und 'Einmischungen' bleiben die konkreten Kirchen mit ihren Traditionen, mit ihren Stärken und Schwächen, ihren Aufbrüchen und typischen Versuchungen." ${ }^{21}$ Eine Gesamtperspektive sah die Redaktion in der ökumenischen Bewegung für ,Gerechtigkeit, Frieden und Bewahrung der Schöpfung'. Nicht alle Leserinnen und Leser konnten sich jedoch mit der ökumenischen Öffnung anfreunden. Einzelne waren der Meinung, der aufbruch müssen ein innerkatholisches Kampfblatt bleiben, mit dem den Tendenzen in der katholischen Kirche entgegengewirkt werden könne.

\subsection{Vernetzung großgeschrieben}

Der aufbruch wollte ausdrücklich die Zeitung einer breiten Basis sein. Diesem Anliegen wurde von Anfang an viel Platz eingeräumt: So finden sich zum Beispiel in den ersten beiden Nummern beeindruckende Listen von ,Mitarbeiterinnen und Mitarbeitern', das heißt Frauen und Männern, die sich bereit erklärt hatten, Beiträge zu verfassen. Ein weiterer Versuch, den aufbruch zu einer Drehscheibe mit vernetzender Kraft werden zu lassen, bildeten die sogenannten ,Leseforen', an denen die Leserinnen und Leser ihre Wünsche zur Sprache bringen, Mängel aufzeigen und zur Klärung von Stil und Profil beitragen konnten. An der ersten ,Aufbruch-Leser-Tagsatzung' im Januar 1989 wurden auch selbstkritische Anmerkungen gemacht: "Wenn der ,Aufbruch" auf längere Sicht gelingen soll (...), dann darf er nicht bloß ,Re-aktion' auf unerfreuliche Entwicklungen in der Kirche sein, sondern muß seine eigene Vision einer ,geschwisterlichen, politischen und mystischen Kirche' in "Aktion' umsetzen." 22 Dies bedeutete, daß der aufbruch nicht bloß der Titel einer Zeitung bleiben durfte, sondern zu einer Basisbewegung werden sollte, die eine starke, spirituelle Herausforderung' in sich birgt und in einer regionalen Vernetzung neue Prozesse auslösen kann. ${ }^{23}$

\section{Verhältnis von ,Zeitung' und ,Bewegung'}

Ein ,Programm' in eine ,Bewegung' umzusetzen, die davon lebt, daß sich Frauen und Männer zu eigenen, konkreten Schritten anregen lassen, ist erfahrungsgemäß ungleich schwieriger als eine Zeitung zu produzieren und dafür Leserinnen und Leser zu finden. Mit dieser Tatsache sah sich auch die Zeitung als bis dahin einziger institutioneller

\footnotetext{
Ebd.

aufbruch Nr. 4, Juli 1989, 1.

aufbruch Nr. 2, März 1989, 2.

Vgl. ebd.
} 
Träger der Idee einer ,Aufbruch-Bewegung' konfrontiert. Nach den Vorstellungen der ,Aufbruch-Pioniere' sollte sich in Anlehnung an die holländische 8.-Mai-Bewegung ein ,Aufbruch-Dachverband' bilden, in dem sich verschiędene Gruppierungen zusammenschließen und dessen internes Organ und Sprachrohr der aufbruch sein würde.

Die Arbeit an der Vernetzung der Leserinnen und Leser wurde Ende 1990 verstärkt an die Hand genommen, nachdem sich die Zeitung weitgehend konsolidiert hatte - der seit Juni 1990 hauptamtlich angestellte Redaktor übernahm einen großen Teil der Arbeit, die zuvor vom ehrenamtlich arbeitenden Redaktionsteam geleistet worden war. Zugleich sollte die Zeitung auf ein juristisches Fundament gestellt werden, das heißt, die Trägerschaft konkretisiert werden. Diese Fragen wurden auf der ersten Generalversammlung des ,Förderkreises aufbruch" im März 1991 diskutiert. Für das Ausarbeiten des Konzeptvorschlages für eine vereinsrechtliche Struktur der Zeitung mit Redaktionsteam, Vorstand und Generalversammlung sowie für die äußere Struktur der Aufbruch-Bewegung mit verschiedenen Arbeitsgruppen, regionalen Gruppen, Kontaktpersonen und einem Koordinationsrat wurde ein sechsköpfiger Vorstand gewählt. Erste regionale und überregionale Gruppen sollten die Aufbruch-Bewegung in der Zeit bis zur nächsten Generalversammlung bereits vorantreiben. Über die Aktivitäten solcher regionaler Treffen konnte man unterschiedlich oft im aufbruch lesen: Einige Gruppen meldeten sich regelmäßig in kurzer Form zu Wort, von anderen hörte man fast nie etwas.

Auf der zweiten ordentlichen Generalversammlung des Vereins „Förderkreis aufbruch" im Januar 1992 wurden die Statuten für den Verein verabschiedet und das Organigramm des ,Aufbruch-Hauses'. mit seinen beiden Säulen, der Zeitung und der Bewegung, vorgestellt. Über die Klärung des Verhältnisses zwischen der Zeitung und der Bewegung erfuhr man jedoch nichts Konkretes, und es wurde wieder ziemlich ruhig um die Bewegung. Einladungen zu regionalen Treffen oder Berichte darüber wurden seltener.

Mehr Erfolg waren den gesamtschweizerischen ökumenischen Aufbruch-Festen beschert. Das erste fand im September 1990 in Olten unter dem Thema ,Damit die Hoffnung nicht stirbt' statt. Über 800 Personen nahmen daran teil. Das zweite lud im September 1993 in die Zentralschweiz nach Brunnen ein. In einem Gesprächsforum mit Vertreterinnen und Vertretern von regionalen Aufbruch-Gruppen kam es dabei zu einer Klärung der Stellung und Situation der auch über fünf Jahre nach den ersten Ereignissen im Bistum Chur noch nicht wunschgemäß in Schwung gekommenen Bewegung.

Anläßlich des Treffens in Brunnen wurde man sich bewußt, daß die Erwartungen, die man von der Aufbruch-Bewegung hatte, teilweise zu groß waren, und daß die anfänglich sehr hoch gesteckten Ziele die Glut der ersten, spontanen ,Aufbrüche' zu ersticken drohten. Einen 
Eindruck von der Situation der Bewegung vermittelt der aufbruchBericht über das Treffen in Brunnen: „Immer wieder wird gefragt, was denn die Aufbruch-Bewegung genauer sei. (...) Worum geht es bei diesen Aufbruchtreffen? Die Antworten der regionalen Verantwortlichen und die Erfahrungen mit dem 2. Aufbruchfest gehen alle in die gleiche Richtung: Gewünscht sind nicht neue, feste Gruppen, eine neue Organisation. Die regionalen wie das nationale Aufbruchtreffen dienen dem Austausch, der gegenseitigen Ermutigung, dem gemeinsamen Feiern. (...) Die Aufbruch-Bewegung ist also nicht, wie sich das anfangs einige vielleicht erhofft hatten, eine fest strukturierte Organisation. Die Aufbruch-Bewegung hat sich auch nicht zu einem Dachverband entwickelt, dem alle möglichen anderen kirchlichen Bewegungen und Organisationen angehören. ${ }^{24}$

Damit stand fest, daß der aufbruch seine Identität nicht mehr über den Status als, Sprachrohr und Drehscheibe einer (noch zu schaffenden) Bewegung' definieren konnte, obschon er den noch bestehenden regionalen Gruppen weiterhin zum Informationsaustausch zur Verfügung stand. Die faktisch schon länger geschaffene, Selbständigkeit' der Zeitung wurde nun auch auf dem Papier bestätigt, wobei das neutralere Umfeld aber auch eine undefiniertere Identität mit sich brachte. Das Fazit lautete schlicht: "Unter dem Stichwort Aufbruch ist zu unterscheiden zwischen der Aufbruch-Zeitung und der Aufbruch-Bewegung. Die Zeitung wird getragen vom Verein ,Förderkreis aufbruch', zu dem Leser und Leserinnen gehören, die den Förderkreis-Beitrag ( $\mathrm{sFr}$ 50,-; Abo inkl.) bezahlen. Der Verein hat einen Vorstand, welcher verantwortlich ist für die Geschäftsführung der Zeitung aufbruch sowie die Anstellung der Redaktorin und des Redaktors. Ein Redaktionsteam ist zusammen mit den beiden Angestellten verantwortlich für die redaktionellen Belange der Zeitung. Die Aufbruch-Bewegung (...) [beabsichtigt, d.Verf.] dem Netzwerk ,Offene Kirche Schweiz' beizutreten (...)." ${ }^{25}$

\section{Nach dem Aufschwung: sinkende Abonnentenzahlen}

Der aufbruch hatte Ende 1991, drei Jahre nach der Lancierung, knapp 10.900 zahlende Abonnentinnen und Abonnenten. Dieser Erfolg und die finanzielle Lage ließen es zu, einen Ausbau der Zeitung ins Auge zu fassen: Auf Januar 1993 wurde die feste Redaktion um eine halke Stelle aufgestockt, und der aufbruch erschien fortan nicht melır nur zweimonatlich, sondern $11 \mathrm{mal} \mathrm{im} \mathrm{Jahr,} \mathrm{jedoch} \mathrm{in} \mathrm{einer} \mathrm{schlankeren}$ Fassung von 12 statt 16 Seiten pro Ausgabe.

Während der Abonnentenstand bis Ende 1992 nur geringfügig auf gut 10.600 zurückging, sackte er in den Jahren 1993 und 1994 drastisch

24 aufbruch Nr. 35, Oktober 1993, 7.

25 aufbruch Nr. 45, September 1994, 6. 
ab: Innerhalb von zwei Jahren verlor der aufbruch rund 2.000 Abonnentinnen und Abonnenten und hatte Ende 1994 noch knapp 8.700. Warum dieser massive Rückgang? Einen ersten Anhaltspunkt bot die Auswertung der schriftlichen, kommentierten Abbestellungen, die der Redaktion 1994 zukamen: $25 \%$ waren inhaltlich begründet: Der aufbruch sei zu links, zu feministisch etc. Ebenso viele Leserinnen und Leser gaben ,technische Fehler' an (erhielten mehrere Exemplare, hätten ihn schon lange abbestellt, hätten ihn noch nie abonniert). Ein gutes Fünftel gab an, keine Zeit mehr zum Lesen zu haben, und für $14 \%$ gab das Alter oder eine Krankheit den Ausschlag. Die übrigen $15 \%$ teilen sich auf in ,finanzielle Schwierigkeiten', ,ins Ausland verreist', ,verstorben' und ,übriges'.26

Der Vorstand des Förderkreises stellte die unangenehme Entwicklung in einen größeren kirchenpolitischen Kontext: "Diese AboEntwicklung läßt sich nur teilweise durch die Erhöhung des Abo-Preises [von sFr 18,- auf sFr 25,- per 01.01.94; d. Verf.] und die wirtschaftliche Rezession erklären. Verschiedene religiös und kirchlich orientierte Zeitschriften haben in den letzten Jahren an Boden verloren. Für viele Menschen dauert der Kirchenwinter nun schon zu lange. Kirchenamtliche Entscheidungen - zuletzt der Fall Gaillot - tragen zur Ausbreitung der Resignation bei. Die damit verbundenen Kirchenaustritte und die innere Emigration vieler Christen und Christinnen schlagen sich natürlich auch in den Abo-Zahlen nieder. ${ }^{\prime 27}$

Im Zusammenhang mit der Frage, wie man dieser Entwicklung entgegenwirken und damit das Weiterbestehen des aufbruch sichern könnte, wurden neben der Erschließung anderer Finanzquellen auch konzeptuelle Veränderungen und damit das Profil der Zeitung diskutiert. Im Ausblick des Vorstandes auf das Jahr 1995 war von einer möglichen, grundlegenden Neuorientierung der Zeitschrift' die Rede: "Zur Diskussion steht insbesondere die heute noch enge Bindung an die Kirchen. Vielleicht ist in den nächsten Jahren eine Ausweitung des Spektrums von Lesern und Leserinnen auf alle religiös suchenden Menschen anzustreben. Auch inhaltlich könnte die Fokussierung auf eine zentrale Thematik, eine Kernidee, dem aufbruch ein neues Gesicht verleihen. Die Zeitschrift ist nicht Selbstzweck. Sie ist vielmehr ein ,Gefäß und soll dieser - noch zu erarbeitenden Kernidee - in der Öffentlichkeit zum Durchbruch verhelfen."28

\footnotetext{
26 Vgl. dazu auch M. Müller, 50 Nummern aufbruch (Anm. 14), $45 f$.

27 aufbruch-Jahresbericht 1994, in: M. Müller, 50 Nummern aufbruch (Anm. 14), Anhang XXVI.

28

Ebd.
} 


\section{Zur 50. Ausgabe des aufbruch: Eine Umfrage unter den Leserinnen und Lesern}

Anläßlich einer Profiltagung im Herbst 1994 faßste der Vorstand und die Redaktion die Durchführung einer Umfrage unter den Leserinnen und Lesern ins Auge. Die Ergebnisse sollten ihre bisher mehr oder weniger gefühlsmäßigen Eindrücke bezüglich der Leserschaft klären oder korrigieren und ihnen damit Anhaltspunkte für ihr weiteres Arbeiten liefern. Konkret sollte die Umfrage in Erfahrung bringen:

a) wie sich die Leserschaft nach Alter, Geschlecht, Konfession etc. zusammensetzt,

b) wie stark ihre kirchliche Bindung und Verbundenheit ist,

c) wie ihr Leseverhalten aussieht,

d) wie der Inhalt und die Aufmachung des aufbruch beurteilt wird und

e) wie die Themeninteressen gelagert sind. Schließlich sollte geklärt werden,

f) wie die Meinungen der Leserinnen und Leser zu verschiedenen Punkten (Konzept, Schwerpunkte, Leitgedanken etc.) gelagert sind.

Um die Umfrage möglichst kostengünstig durchführen zu können, wurde dem aufbruch vom Februar 1995, der sogenannten "Jubiläumsnummer 50", ein Fragebogen beigelegt. Von den gut 10.500 versandten Fragebogen wurden 1885 der Redaktion zurückgesandt, was einem Rücklauf von $18 \%$ entspricht. Bezogen auf die festen Abonnements (gut 8.800 ) erreicht er sogar $21 \% .^{29}$

\section{3. Überraschende und klärende Ergebnisse}

\subsection{Struktur der Leserschaft}

Die aufbruch-Leserschaft setzt sich gemäß den Ergebnissen der Umfrage aus $51 \%$ Frauen und $49 \%$ Männern zusammen, von denen $82 \%$ römisch-katholisch und $14 \%$ evangelisch-reformiert sind. Gut $2 \%$ sind konfessionslos. Auffällig häufig wurde auf dem Fragebogen von der Option ,römisch-katholisch' der Teil ,römisch" gestrichen, was als deutlicher Hinweis für die Identifikation der LeserInnen mit der kritischen Haltung des aufbruch gegenüber der offiziellen Kirchenleitung gesehen werden kann.

Die Altersstruktur überraschte auf den ersten Blick: Fast $70 \%$ der Leserinnen und Leser sind 45jährig und älter, wobei alleine $22 \%$ 65jährig und älter sind. Die 25-34jährigen sind mit lediglich $11 \%$

$29 \mathrm{Zu}$ den technischen Angaben über die Vorbereitung und Durchführung sowie zu Problemdiskussionen bezüglich der Auswertung der Umfrage siehe M. Müller, 50 Nummern aufbruch (Anm. 14), 49-63. 
vertreten. Auch unter Berücksichtigung eines Unschärfefaktors, der davon herrühren könnte, daß die älteren Leserinnen und Leser eher Zeit hatten, den (umfangreichen) Fragebogen auszufüllen, zeigt sich doch ganz deutlich, daß der aufbruch primär ältere Menschen anspricht. Diese Altersstruktur wird sich in den nächsten Jahren in verstärktem Maße negativ in der Abonnentenzahl bemerkbar machen, wenn es nicht gelingt, neue Abonnentinnen und Abonnenten zu finden, die gemäß der Auswertung tendenziell jünger werden.

Weniger überraschend wirkt diese Altersstruktur im Wissen, daß $34 \%$ der aufbruch-LeserInnen im theologischen, kirchlichen oder karitativen Bereich erwerbstätig sind, und daß die übrigen Leserinnen und Leser eine sehr starke kirchliche Verbundenheit aufweisen: Fast 60 $\%$ von ihnen besuchen mindestens alle 14 Tage einen Gottesdienst und $43 \%$ sind Mitglied einer kirchlichen Gruppierung. ${ }^{30}$ Damit entspricht die Altersstruktur der aufbruch-Leserschaft in etwa jener der allgemein in Pfarreien und Kirchgemeinden Aktiven und Engagierten. Im weiteren ist erwähnenswert, daß die stark vertretene Altersgruppe der über 45jährigen jenem Segment von Frauen und Männern entspricht, die das ,aggiornamento' des Zweiten Vatikanischen Konzils bewußt miterlebt haben, darin aufgewachsen sind und sich mit diesem Prozeß persönlich auseinandersetzen mußten. Der aufbruch kann besonders für sie in einer Zeit restaurativer Entwicklungen innerhalb der Kirche ein Identifikationsmedium für ihren gelebten Glauben darstellen. Dies wurde vereinzelt auch in qualitativen Bemerkungen im Rahmen der Umfrage zum Ausdruck gebracht.

$\mathrm{Da}$ die Leserschaft überaus stark kirchlich interessiert ist, wird nicht zuletzt durch die Auswertung der Frage bestätigt, worüber sich die LeserInnen mit dem aufbruch identifizieren. Die Antwortmöglichkeiten deckten die drei Schwerpunkte des aufbruch ab: Kirche/Kirchenpolitik, Religion und Gesellschaftspolitik. Zusätzlich wurde die Option angeboten, der aufbruch sei eine Art ,Mitgliedszeitschrift' der AufbruchBewegung. Aus den Kombinationen der Antworten (Mehrfachantworten waren möglich) läßt sich schließen, daß die Identifikation primär über die kirchliche und religiöse Ausrichtung des aufbruch läuft. Am häufigsten genannt wurde: ,Er liefert Hintergrundinformationen über die Entwicklungen in den Kirchen' (69\%). Das Interesse für Religion und Kirche läßt sich bei einem nicht zu vernachlässigenden Anteil

30 Die extrem starke kirchliche Bindung wird noch deutlicher im Vergleich mit zwei Studien, einer Umfrage unter den LeserInnen des Pfarrblattes des Kantons Zürich ,forum' (vgl. LINK-Leserschaftsbefragung ,forum' 3.0688, Luzern 1992), wo $44 \%$ der LeserInnen mindestens 14-täglich einen Gottesdienst besuchen, und einer gesamtschweizerischen religionssoziologischen Studie (vgl. A. Dubach/R. J. Campiche (Hrsg.), Jede(r) ein Sonderfall. Religion in der Schweiz, Zürich 1993, 340), bei der noch $26 \%$ eine entsprechende Antwort gaben. 
LeserInnen nur bedingt mit einem Interesse für die Aufbruch-Bewegung in Beziehung bringen, deren Bekanntheitsgrad unter der Leserschaft entsprechend ihrer schwindenden Konkretheit über die Jahre abgenommen hat. Die Identifikation über gesellschaftspolitische Themen' ist deutlich schwächer (39\%).

Bei der klassischen ,Vermissensfrage' gaben $85 \%$ an, sie würden den aufbruch vermissen, wenn es ihn nicht mehr gäbe. Diese Zahl setzt sich zusammen aus $25 \%$, die ihn ,sehr' und $60 \%$, die ihn ,eher schon' vermissen würden. Der aufbruch hat demnach ein äußerst interessiertes Publikum. Von jenen LeserInnen, die die Zeitung kaum (11\%) oder überhaupt nicht ( $1 \%$ ) vermissen würden, gaben mehrere einen Grund an wie ,sonst noch genug zu lesen': aufbruch-LeserInnen lesen durchschnittlich 3,6 andere religiöse oder kirchliche Publikationen.

Aus den Angaben, welche Publikationen neben dem aufbruch noch gelesen werden, lassen sich einerseits bisher aufgezeigte Punkte verdeutlichen und andererseits neue typische Merkmale der aufbruchLeserInnen aufzeigen: Die starke kirchliche Verbundenheit der Leserinnen und Leser zeigt sich zusätzlich im bestechend hohen Prozentsatz von $88 \%$, die das Informationsorgan ihrer Pfarrei oder Kirchengemeinde abonniert haben, bzw. lesen. Dazu lesen über $50 \%$ neben dem aufbruch den 'Wendekreis', eine der ältesten und etabliertesten katholischen Publikationen in der Schweiz. 31 \% lesen die ökumenische, von Frauen geschriebene Zeitschrift ,Schritte ins Offene, die "Themen aus dem Bereich Emanzipation-Glaube-Kulturkritik behandelt und sich an Christinnen und Christen richtet, die in der Kirche verwurzelt sind, und solche, die der Kirche kritisch gegenüberstehen ". ${ }^{11}$ Schließlich überrascht vor allem die Zeitschrift ,Orientierung", die von $25 \%$ der aufbruch-LeserInnen gelesen wird und allgemein als eher anspruchsvolle, ,elitäre‘ Publikation gilt. 11 \% lesen ,Publik Forum‘.

Die daraus ableitbare Vermutung, daß der aufbruch eine eher überdurchschnittlich gebildete Leserschaft hat, wird durch die Auswertung der Frage nach der Ausbildung bestätigt: Ihr zufolge haben $37 \%$ ein abgeschlossenes Hochschulstudium, dazu $26 \%$ eine höhere Berufsschule. ,Nur' $23 \%$ gaben an, eine Berufsschule bzw. -lehre absolviert zu haben. Neben dem großen Anteil akademisch gebildeter Theologinnen und Theologen unter der Leserschaft weisen auch die ,übrigen', nicht kirchlich erwerbstätigen Leserinnen und Leser eine vergleichsweise überdurchschnittliche Bildung auf. ${ }^{32}$

31 Vgl. die Kurzportäts der im Fragebogen aufgeführten kirchlichen und religiösen Publikationen, in: M. Müller, 50 Nummern aufbruch (Anm. 14), Anhang Il-VII.

32 Vgl. dazu auch A. Dubach/R. J. Campiche (Hrsg.), Jede(r) ein Sonderfall (Anm. 30), 341. Diese Studie stellt einen repräsentativen Querschnitt durch die schweizerische Bevölkerung dar. 


\subsection{Inhaltliche Ausrichtung}

Als das zentrale Interesse und Anliegen der Leserinnen und Leser hat sich in der Auswertung der Umfrage das Thema ,Ökumene' herausgestellt: In einer Bewertung der Schwerpunktthemen der Jahre 1993 und 1994 wurde die Nummer mit dem Titel ,Gelebte Ökumene' als Spitzenreiter von $54 \%$ der Leserinnen und Leser als, sehr interessant und von $32 \%$ als ,ziemlich interessant ' beurteilt. Unabhängig davon, ob sie sich noch an den konkreten Inhalt der entsprechenden Nummer erinnern konnten oder nicht, unterstrichen die Leserinnen und Leser dazu, daß der aufbruch eine ökumenische Zeitung sein und bleiben soll: Der Aussage ,Mir ist wichtig, daß das ökumenische Anliegen spürbar ist' stimmten $67 \%$, voll und ganz' und $26 \%$,eher' zu.

Im Kreuzvergleich der beiden Fragen kommt zum Ausdruck, daß für $83 \%$ der LeserInnen die Ökumene ein zentrales Thema ist. Nur knapp $2 \%$ können damit explizit überhaupt nichts anfangen.

Aus der Rangliste der Schwerpunktthemen (von ,Gelebte Ökumene' und "Rassismus' über ,Feministische Theologie' und "Kinderrechte' bis ,Ordensleben' und ,IWF/Weltbank'), lassen sich keine eindeutigen Präferenzen für kirchliche oder gesellschaftspolitische Themen ausmachen - entgegen dem deutlich verringerten Grad an expliziter Identifikation mit der Zeitung über das gesellschaftspolitische Engagement. Es scheint den LeserInnen demzufolge weniger darauf anzukommen, aus welchem Bereich das Schwerpunktthema stammt, als vielmehr um die Art und Weise, wie es aufbereitet und diskutiert wird. Dies spricht einerseits für die kritische Lektüre der LeserInnen, andererseits zeigt es aber auch, daß die Redaktion das sonst eher kirchlich geprägte Interesse der Leserschaft mit interessanten gesellschaftpolitischen Nummern auf diesen Themenzweig lenken kann.

\section{Wo steht der aufbruch heute?}

Der aufbruch hat sich am Anfang seines Auftretens 1988 ein klares Profil mit klaren Optionen gegeben: Er hat sich als Ziel gesetzt, eine Zeitung der ,Basis' zu sein, als ein kritisches Medium nicht nur zu reagieren, sondern auch neue Visionen aufzubauen und zwar aus einer ökumenischen Gesamtperspektive. Diese Optionen, die eine Antwort auf krasse Mißstände in der ,Kirche Schweiz' sein wollten, trafen - nicht zuletzt wegen der damals aktuellen Situation - auf eine breite Resonanz. Die Entwicklungen im kirchlichen Umfeld und in der Aufbruch-Bewegung haben jedoch in den folgenden Jahren dazu geführt, daß der aufbruch seine Identität immer wieder neu definieren mußte: Spätestens seit der Ernennung der beiden Weihbischöfe Vollmar und Henrici, die im Jahre 1993 dem umstrittenen Diözesanbischof von Chur zur Seite gestellt worden waren, ist es um diesen gespenstisch still geworden, und die zunächst so hoffnungsvoll initiierte Aufbruch-Bewegung lebt nur noch 
in vereinzelten Gruppen weiter. Der aufbruch von 1996 ist nicht mehr der aufbruch aus den späten 80er Jahren. Wo steht der aufbruch mit seinen Optionen heute?

Viele Leserinnen und Leser haben das Jubiläum im Februar 1995 und die damit verbundene Umfrage zum Anlaß genommen, der Redaktion auch über den Fragebogen hinaus ein kurzes Feedback zu geben. Der Vergleich der oft sehr persönlich geprägten Äußerungen brachte deutlich zum Ausdruck, daß die Ansichten der Leserinnen und Leser, was der aufbruch sein sollte und was er nicht sein darf, so stark divergieren, daß es fast unmöglich scheinen muß, alle Vorstellungen in einem Profil zusammenzufassen: Den einen ist der aufbruch zu ökumenisch oder zu gesellschaftspolitisch. Sie möchten ihn am liebsten als ein katholisches ,Kampfblatt' sehen und sprechen ihm eine Art Beobachterstatus in der Kirche zu. Andere finden es hingegen grundsätzlich müßig, sich überhaupt noch mit der Institution der (katholischen) Kirche auseinandersetzen und möchten vor allem den allgemeinen christlich-kritischen Blick auf die Gesellschaft verstärkt wissen. Der größte Teil der Leserinnen und Leser steht irgendwo zwischen diesen beiden Extrempositionen. Es sind Frauen und Männer, die die Kirche als Heimat suchen, sie als solche brauchen und gleichzeitig bereit und interessiert sind, sie mitzugestalten. Charakteristisch für sie ist (noch) die Verbundenheit mit der Kirche. Diese ist geprägt von einer kritischen Haltung, die einerseits die Grenzen der starren Institution klar sieht und andererseits die Hoffnung noch nicht aufgegeben hat, daß sich - durch Initiativen von unten und das Engagement an Ort - etwas verändern kann. In diesem Sinn ist der aufbruch für viele Leserinnen und Leser gerade im winterlichen Kirchenklima ein Stück Hoffnung geworden, wie es diese beiden Stimmen im Rahmen der Umfrage ausdrücken: "Der aufbruch macht mir Mut, mich weiterhin für eine geschwisterliche und offene Kirche einzusetzen!" und "Wir sind froh und dankbar, daß es den aufbruch gibt, sonst wären wir an dieser Kirche schon längst verzweifelt! ‘33

Der aufbruch hat sich seit seinem Auftreten innerhalb der Strukturen der ,Kirche Schweiz' zu einem relevanten Medium und einer nicht mehr überhörbaren Stimme entwickelt. Die Umfrage hat gezeigt, daß das aktuelle Zielpublikum nicht, wie eigentlich angestrebt, die ,kirchliche Basis' in einem engeren Sinn ist, d.h. das ,gemeine kirchliche Fußvolk' bzw. die durchschnittlichen Kirchgängerinnen und -gänger. Der aufbruch hat nicht schon durch seinen Namen, der eine Dynamik von unten impliziert, den Charakter eines solchen Mediums. Vielmehr muß der Begriff ,Basis' in einem weiteren Sinn als ,lebendige Kirche am Ort' (hier Pfarrei/Kirchgemeinde) definiert werden. Durch die vielen kirchlich erwerbstätigen Abonnentinnen und Abonnenten sowie die ehrenamtlich engagierten Leserinnen und Leser ist er aber trotzdem in

33 Zit.n. M. Müller, 50 Nummern aufbruch (Anm. 14), 146. 
der (katholischen) Kirche sehr stark verankert. Dieses ,Potential' gilt es für sein Anliegen, seine Vision einer offenen und geschwisterlichen Kirche, möglichst effizient zu nutzen.

Das zweite große Anliegen des aufbruch ist seine ökumenische Ausrichtung, und diese deckt sich, wie gezeigt, mit dem Interesse der Leserschaft. Aus der Umfrage läßt sich jedoch nicht genau erschließen, welche konkreten Vorstellungen die einzelnen Leserinnen und Leser mit dem Begriff ,Ökumene' verbinden: Ist es eine wohlwollende Sicht auf die anderen christlichen Konfessionen, ohne die Bereitschaft, aus dem eigenen Unterschlupf hervorzutreten? Oder stimmt ihre Vorstellung mit derjenigen der Redaktion überein, die einen Orientierungspunkt in der Bewegung für ,Frieden, Gerechtigkeit und Bewahrung der Schöpfung sieht? Wenn es dem aufbruch gelingt, dieses ökumenische Programm neu zu beleben, und zwar in Form eines eigenständigen Sichdaran-Orientierens und Ausarbeitens von konkreten Handlungsperspektiven, die auf verschiedenen Ebenen - individuelles, gesellschaftliches, kirchliches und politisches Leben - vom biblischen Auftrag getragen und motiviert sind, so könnte er wohl auch einen breiteren Kreis von evangelisch-reformierten Frauen und Männer ansprechen und mit ihnen die bisher erst in Ansätzen ökumenische Leserschaftsstruktur noch ökumenischer prägen.

\section{Zwischen ,Option' und ,Erfolg'}

Daß die jüngste Neuorientierungsphase des aufbruch durch die sinkenden Abonnentenzahlen ausgelöst wurde, macht einmal mehr deutlich, daß es im Zweifelsfall nicht die ideellen, sondern oft die ökonomischen Faktoren sind, die die Entwicklung einer Zeitung bestimmen und die ersten spürbaren Konsequenzen nach sich ziehen. So auch beim aufbruch: Trotz aller Anstrengungen mußte der Vorstand eine Ende 1994 noch als ,ultima ratio' bezeichnete mögliche Maßnnahme in der Zwischenzeit in die Tat umsetzen: Der aufbruch erscheint seit Anfang 1996 nur noch acht- statt 11mal jährlich.

Die Situation des aufbruchs stellt keinen Sonderfall dar. Die sich verändernden sozio-religiösen und wirtschaftlichen Faktoren stellen gerade im Bereich der kirchlichen bzw. konfessionellen Presse immer mehr Publikationen vor die Frage nach dem Überleben. Vor allem jüngere Publikationen mit kleinen Auflagen sind gefährdet: Sie müssen sich in einer Zeit zu etablieren versuchen, in der sie mit ihrer konfessionellen - oder im weitesten Sinn christlichen - Prägung oft nur als Nischenprodukt auftreten können. "Erfolg' kann dabei nur ein relativer Begriff bleiben. Die Publikationen müssen sich letztendlich darauf konzentrieren, weiterbestehen zu können, und zwar - soweit möglich - ohne das eingeschlagene Profil aufgeben zu müssen.

Oftmals haben gerade diese Publikationen für ihr Profil Optionen 
getroffen, die Zeugen einer engagierten Auseinandersetzung mit unserer Zeit sind, die jedoch in ihrem Umfeld nur ein beschränkt etabliertes Fundament als Stütze finden können. Erschwert wird ihre journalistische Tätigkeit dadurch, daß bei vielen Leserinnen und Lesern der allgemeine, privat-religiöse Bereich und/oder der kritische Blick auf die kirchenpolitischen Vorgänge in der (katholischen) Kirche emotional äußerst empfindlich oder unter Umständen sogar verletzt ist. $\mathrm{Zu}$ den Publikationen, die diese erschwerten Vorbedingungen meistern müssen, gehört auch der aufbruch. Die beachtliche Resonanz, die allgemein starke Leserbindung und nicht zuletzt der hohe Grad an beruflichem oder ehrenamtlichem kirchlichem Engagement der Leserinnen und Leser zeigen jedoch, daß er sein Anliegen - allen negativen Entwicklungen zum Trotz - aus einem weit verbreiteten Bewußtsein heraus formulieren kann: Sein Engagement ist geleitet von einem elementaren, geschichtlichen und verantwortenden ökumenischen Glauben, der die Entwicklungen in Kirche und Gesellschaft ernst nimmt. Dazu gehört auch eine konsequente Orientierung an einer geschwisterlichen und mündigen Kirche, die sich unter anderem durch eine offene und kritische Kommunikationskultur auszeichnet.

Der Vers "Ich nenne euch nicht mehr Knechte ..." erhält in diesem Kontext den Charakter einer notwendigen, grundlegenden Forderung für eine angstfreie kirchliche Kommunikationskultur, die Platz läßt für neue ,Aufbrüche' im prophetisch-biblischen Sinn; eine Option, die jetzt schon von vielen Seiten mit kleinen, konkreten Handlungen gefördert wird, und an der sich auch der aufbruch beteiligt.

SUMMARY: "aufbruch“ - Forum for a free-minded Church. A new ecumenical magazine searching its identity between the tension of ,option' and success'

The article deals with the magazine "Aufbruch - Forum für eine offene Kirche" (meaning: "Start - forum for a free-minded Church) which was founded 1988 in Switzerland. The magazine was started in connection with the conflict on the appointment and installing of Wolfgang Haas as bishop of Chur. In the first part of the article the author describes the development of the so-called "Case Haas" and demonstrates by this concrete example two types of inner-church communication which are contrary to each other. In the second part the foundation and development of the magazine are described including some perspectives for its further development and the necessary search for a financial, success'.

RÉSUMÉ: "aufbruch“ - Forum pour une église ouverte. Recherche d'identité d'un jeune journal oecuménique qui cherche sa voie entre l'option et la réussite

L'article présente la revue „Aufbruch (Nouveau Départ) - Forum pour une église ouverte" qui a été créé en Suisse en 1988. La revue est née dans le contexte de discussions religieuses à propos de l'investiture de Wolfgang Haas 
comm évêque de Chur. Dans un premier temps, l'auteur décrit le soi-disant „cas Haas" et identifie grâce à cet exemple concret deux types contraires de communication religieuse interne. Dans un deuxième temps, il est question de la création et de l'évolution de la revue "Aufbruch" et du développement de quelques perspectives pour son évolution à venir entre son "option" religieuse et la recherche nécessaire d'une réussite financielle.

RESUMEN: aufbruch - Forum por una iglesia abierta. Búsqueda de la identidad de un periódico ecuménico jóven en la tensión entre opción y éxito

El artículo presenta la revista „aufbruch - Forum por una iglesia abierta” fundada en 1988 en Suiza. La revista surgió en el contexto de la discussión que se produjo en la iglesia en torno al nombramiento de Wolfgang Haas como Obispo de Chur. En la primera parte el autor describe el llamado "Caso Haas" e identifica, partiendo de este ejemplo concreto, dos tipos contrarios de comunicación interna en la iglesia. En la segunda se presentan el nacimiento y el desarrollo de la revista "aufbruch" y se revelan algunas perspectivas para su desarrollo entre la "opción" de iglesia y la necesaria búsqueda de un "éxito" financiero. 